\title{
Dust Storm Detection Using a Neural Network with Uncertainty and Ambiguity Output Analysis
}

\author{
Mario I. Chacon-Murguía ${ }^{1}$, Yearim Quezada-Holguín ${ }^{1}$, \\ Pablo Rivas-Perea ${ }^{2}$, and Sergio Cabrera ${ }^{2}$ \\ ${ }^{1}$ DSP \& Vision Laboratory, Chihuahua Institute of Technology, Chihuahua, Mexico \\ mchaconaitchihuhaua. edu.mx \\ ${ }^{2}$ ECE, University of Texas at El Paso, USA \\ privas@miners.utep.edu, sergioc@utep.edu
}

\begin{abstract}
Dust storms are meteorological phenomena that may affect human life. Therefore, it is of great interest to work towards the development of a stand-alone dust storm detection system that may help to prevent and/or counteract its negative effects. This work proposes a dust storm detection system based on an Artificial Neural Network, ANN. The ANN is designed to identify not just dust storm areas but also vegetation and soil. The proposed ANN works on information obtained from multispectral images acquired with the Moderate Resolution Imaging Spectroradiometer (MODIS) instrument. Before the multispectral information is fed to the ANN a process to remove cloud regions from images is performed in order to reduce the computational burden. A method to manage undefined and ambiguous ANN outputs is also proposed in the paper which significantly reduces the false positives rate. Results of this research present a suitable performance at detecting the dust storm events.
\end{abstract}

Keywords: Dust storm detection, image segmentation, neural network output analysis.

\section{Introduction}

In recent years weather conditions have attracted the attention of the international community. Different countries have suffered the consequences of natural phenomena like earthquakes, tsunamis, floods, hurricane, drought, dust storms, which significantly affect human life as well as the economy of those countries. These climate situations have promoted a new interest on remote sensing research because it offers the potential of better understanding of these phenomena. One of these natural phenomena that have been studied in recent years is dust storm. Dust storms are seasonal meteorological phenomena. Usually, dust storms occur in arid and semiarid regions and can travel long distances. The dust storm may have a negative effect in our planet and human life in different ways like; cloud formation, respiratory illness, aerial and terrestrial transportation. This phenomenon can also damage crops and may cause fertile soil erosion [1]-[3]. Research on dust storm is of great interest because it can help to find methods to prevent and/or counteract its negative effects. This paper 
presents the development of an Artificial Neural Network, ANN, classifier intended to detect the presence of a dust storm in satellite multispectral images. The paper is organized in the following sections. Section 2 presents a literature review of this topic. The dust storm data used in this work is described in Section 3. Analysis of the classifier is covered in Section 4. Finally the results and conclusions of the research are commented in Section 5.

\section{Literature Review}

In order to establish some aspects of the classifier design, a literature review was achieved considering the following points: type of instrument used to acquire the multispectral images, methods used for dust storm detection, performance metrics, and event location. From 43 papers related to the topic of dust storm and satellite multispectral images only 13 were considered for the analysis because only these papers were related specifically to dust storm detection. With respect the type of sensor used, works reported in [4] to [9] use the Moderate-Resolution Imaging Spectroradiometer instrument, commonly named MODIS sensor. The researches in [6] and [7] work with the Aqua MODIS. The sensor AERONET and the AVHRR are used in [10] and [11] respectively. The work reported in [12] employs the CALIPSO and in [13] the MERIS sensor is used to detect dust storm in sand regions. The MFR7 sensor is mentioned in [14]. Also in [9] it is reported a combination of information obtained from the sensors MODIS and TOMS. Finally, the MISR sensor is used in [15]. With regard to the type of image processing techniques used, the information is the following. All works are based on pixel level feature extraction processing, except in [9] and [10] where a window feature extraction processing is used instead. The method used to evaluate the performance of the dust storm detection systems was only qualitative. The dust storm events reported in the literature were located at; South Korea, China, Mongolia, India, Egypt, Senegal and East of Africa. In some of these works it was necessary to adjust some of the parameters of the method developed in order to obtain correct results.

Considering the information found in the literature review the following points were determined. It was decided to use the MODIS sensor because it has a good spectral resolution, 36 bands from $0.62 \mu \mathrm{m}$ to $14.382 \mu \mathrm{m}$. The temporal resolution is 15 minutes and its spatial resolution $1 \mathrm{Km}$. The image processing technique selected was pixel level processing because most of the reported works that used this technique achieved better results than the two papers that used window based processing.

\section{Data Analysis}

\subsection{Database}

The region of interest for dust storm detection is defined as the north region of Chihuahua State in Mexico and the southwest area of Texas State in the USA. The dust storm events were acquired from the web page http:// ladsweb.nascom.nasa.gov/ data/ search.html. These events were acquired by the MODIS sensor. Eight events were downloaded and their information is given in Table 1. 
Table 1. Dust Storm Event Information

\begin{tabular}{|c|c|c|c|}
\hline Date & Hour & Date & Hour \\
\hline 1. April/6/2001 & 18:30 hrs. & 5. April/15/2003 & 17:10 hrs. \\
\hline 2. April/10/2001 & 18:05 hrs. & 6. April/15/2003 & $17: 15 \mathrm{hrs}$. \\
\hline 3. July/2/2002 & $17: 55 \mathrm{hrs}$. & 7. April/15/2003 & 18:50 hrs. \\
\hline 4. December/17/2002 & $18: 45$ hrs. & 8. November/22/2003 & $18: 20 \mathrm{hrs}$. \\
\hline
\end{tabular}

\subsection{Band Selection}

Not all the information in the bands of the multispectral images is related to dust storm events. In order to determine which bands will be used in the design of the classifier a literature review was achieved. The bands B31 and B32 of the MODIS sensor were related to dust storm information in works reported in [5]-[9]. The bands 4 and 5 of the sensor AVHRR were used for dust storm detection in [1] and [2] but these bands correspond to the bands 31 and 32 of the MODIS sensor. In [6] the band B29 of the MODIS was incorporated because it provides extra information in clear days. Based on these evidences we concluded to use the bands B29, B31 and B32.

\subsection{Data Selection}

In order to obtain reliable samples for the neural network design, data was statistically selected for each class. The classes considered in this work were dust storm $D$, vegetation $V$, and soil $S$. The samples selected correspond to samples obtained from regions of each class that satisfy the following criterion

$$
D_{p_{c}} \leq D_{c} \quad \text { for } \quad c=\{V, D, S\} .
$$

where $D_{p c}$ is the distance of the candidate sample $p_{B c}$ of the band $B$ of the region $c$ to the mean value of the sample mean, $\mu_{B n c}$, of the band $B$ in the region $c$,

$$
D_{p_{c}}=\sqrt{\left(\mu_{B 1_{c}}-p_{B 1 c}\right)^{2}+\cdots+\left(\mu_{B n c}-p_{B n c}\right)^{2}} .
$$

$D_{c}$ is the maximum tolerance distance, one standard deviation $\sigma_{B n c}$, of a sample of a region $c$ to the sample mean of the region.

\section{Dust Storm Detection}

This section describes the process of the ANN classifier design including a step to detect clouds present in the images and then it follows with the design of the ANN classifier.

\subsection{Cloud Detection}

Multispectral images in most of the cases represent huge computational burden, therefore it is recommended to reduce the amount of information as much as possible. It was reported in the literature [6], that presence of clouds is an important perturbation that affects the performance of several dust storm detection methods. Thus, cloud detection is 
a good alternative to reduce the computation load of dust detection algorithms and at the same time to get rid of possible perturbations. The work in [4] reports that cloud information can be eliminated using bands $B_{3}$ and $B_{7}$. The work shows that the maximum energy related to clouds is captured by band $B_{3}$. Meanwhile energy of other elements is minimum in this band. Band $B_{7}$, contrary to band $B_{3}$, capture minimum energy related to clouds and high energy the other elements. Based on the previous information, it is proposed a difference index $I_{D}=B_{7}-B_{3}$ to eliminate cloud information. Using this index a mask image, $I_{M}$, can be generated to eliminate the cloud pixels.

$$
\mathrm{I}_{M}=\left\{\begin{array}{lll}
0 & \text { if } & I_{D}<0 \\
1 & \text { if } & I_{D}<0
\end{array} .\right.
$$

Other important result is that the $I_{D}$ of water pixels will result in zero or near to zero values. Therefore water pixels will be also eliminated. The application of this process is illustrated in Figure 1, event \#1 April/6/2001. The original information is shown in Figure 1a, the mask image is shown in Figure 1b and the resulting information of the event is shown in Figure 1c. The cloud elimination process presents some issues. On one hand, small clouds are not completely eliminated. On the other hand, some dark vegetation and soil regions are eliminated. However dust storm information is not significantly affected by this process. An example of this screening is shown in Figures 1d-f.

\subsection{Classifiers Design}

The classifier was designed to recognize three classes: vegetation, dust storm and soil; unlike other works that only consider a two-class problem dust storm and not dust storm. It was decided to include the class vegetation and soil because they may be of interest in a future work.

The structure of the neural network was determined by selecting the best network in a set of experiments changing the number of layers, 2 and 3 layers, and the number of neurons in each layer. The best neural network was defined with respect the best performance associated with the smaller number of layers and neurons by layer. The
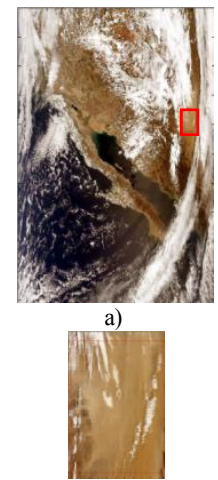

d)
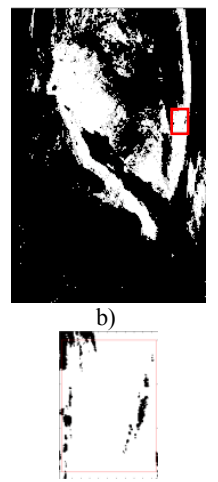

e)
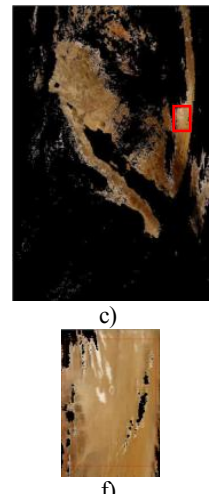

f)

Fig. 1. a) Original image event April/6/2001, b) $I_{M}$ mask, and c) Image without clouds and water. d), e) and f) zoom-in of the red rectangles in a), b) and c) 
ANN model selected for the classifier is a 2-layer Feed-Forward neural network with 15 neurons in the hidden layer, and 3 neuron outputs. The activation functions are sigmoidal. The training was performed with the backpropagation algorithm using the Scaled Conjugate Gradient Algorithm since this algorithm was more effective than other gradient methods. Among their advantages we can mention that it does not depend on user parameters like learning rate and momentum. The first classifier, C29, was designed with the information provided by the bands B29, B31 and B32. The number of samples used was 5383; 721 samples of vegetation, 1493 of dust storm and 3169 of soil. The training samples correspond to the $70 \%$ of the total number of samples, $15 \%$ for validation and $15 \%$ for testing. The performances of this ANN were $95.8 \%$, of correct classification for training, validation and testing. Table 2 shows the confusion matrix of the design process. It can be observed that the class dust storm has the best classification performance considering actual and prediction conditions $97.45 \%$ followed by Soil, $96.95 \%$ and vegetation $89.4 \%$. The total performance is $96 \%$. Figure 2 shows some visual results obtained with this classifier for the events \#2, and 8, corresponding to April/10/2001, and November/22/2003 respectively. The result of the event April/10/2001 presents some false positives areas close to the dust storm region. The event April/15/2003 shows a correct detection, but the event of November/22/2003 involves many false positives regions.

Results on non-storm events are not shown because the work is related to the same area; therefore data of these cases is already incorporated and tested on the different dust storm events.

Table 2. Confusion matrix of the classifier C29

\begin{tabular}{lllll}
\hline & Vegetation & Dust Storm & Soil & Total \\
\hline Vegetation & 578 & 0 & 8 & $98.6 \%$ \\
Dust Storm & 12 & 1450 & 20 & $97.8 \%$ \\
Soil & 131 & 43 & 3141 & $94.8 \%$ \\
Total & $80.2 \%$ & $97.1 \%$ & $99.1 \%$ & $96 \%$ \\
\hline
\end{tabular}
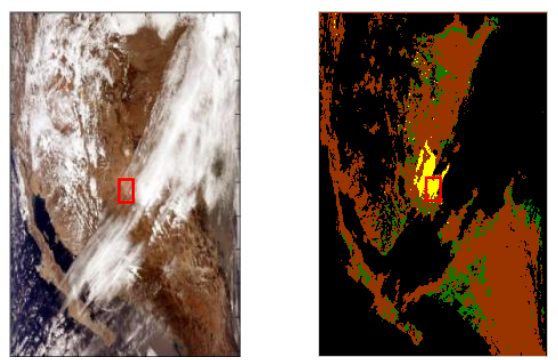

a)

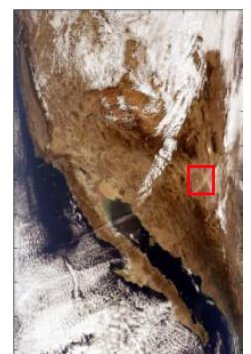

b)

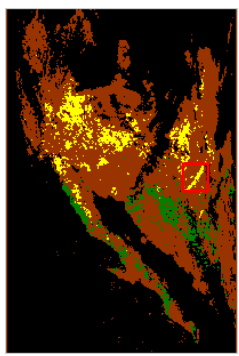

Fig. 2. Results of the classifier C29 for the events: a) April/10/2001, b) November/22/2003. Cloud /water black, vegetation green, storm yellow, soil brown 


\subsection{C29 Output Analysis}

The results shown in the previous section led us to investigate the neuron outputs. The previous results were generated by the following rule Assign pixel $p_{B}$ to class if if $O_{i}>$ $O_{j}$ for $i, j=\{v, d, s\} i \neq j$, where $v, d$, and $s$ stands for the classes vegetation, dust storm and soil respectively. This rule assigns a pixel to the class with the higher value of the neuron outputs. This kind of decision is generally used in some works. However, this is not a guarantee that a good decision is taken. We can analyze two hypotheses: the first one supposes that three or two neuron outputs are high but both with close values; the second hypotheses could be when all outputs are low. In both cases we can determine a winner output but it does not mean the decision will be correct. From this analysis we can propose two cases. An undefined case, when the maximum output of the ANN is less than say 0.6, that is

$$
\max \left(O_{i}<0.6\right) \text { for } i=\{v, d, s\} .
$$

In this situation we can say that the ANN does not have a strong output in any of its outputs, therefore the class is undefined. The 0.6 threshold was determined by considering the output of the neurons as an effectiveness class definition percentage, the value 0.5 represents high vagueness. The second case is when the difference between two neuron outputs used to make a decision is less than 0.3 ,

$$
\left|O_{i}-O_{j}\right|<0.3 \text { for } i=\operatorname{argMax} O_{K} \text { and } j=\operatorname{argMax}_{K} \quad k=\{v, d, s\} i \neq j .
$$

In this circumstance, the outputs of the ANN are so ambiguous that a decision is not recommended. Using the previous cases two new images can be generated to analyze the two hypotheses, $I_{u}(x, y)$ for undefined outputs and $I_{A}(x, y)$ for ambiguous outputs

$$
I_{u}(x, y)=\left\{p_{B}(x, y): \max \left(O_{i}\right)<0.6 \text { for } i=\{v, d, s\}\right\} .
$$

$I_{A}(x, y)=\left\{p_{B}(x, y):\left|O_{i}-O_{j}\right|<0.3\right.$ for $i=\operatorname{argMax}_{K}$ and $\left.j=\operatorname{argMax}_{K} \quad k=\{v, d, s\} i \neq j\right\}$.
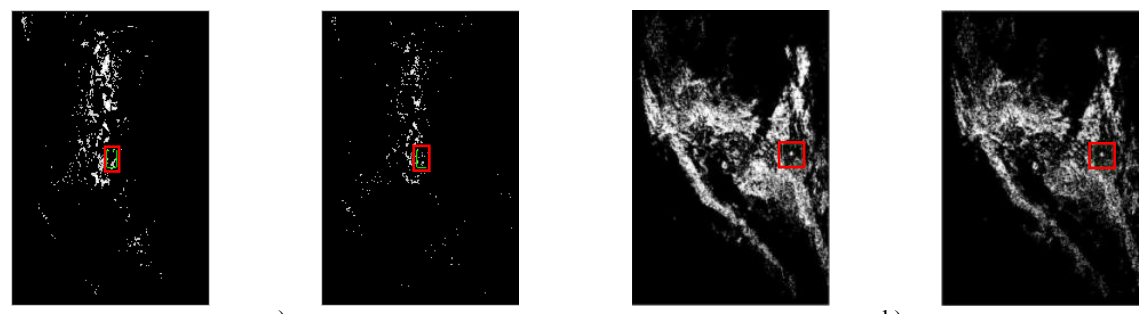

a)

b)

Fig. 3. $p(x, y) \in I_{u}(x, y)$ and $p(x, y) \in I_{A}(x, y)$ of a) April/10/2001, b) November/22/2003 
Figure 3 illustrate the pixels $p(x, y) \in I_{u}(x, y)$ and $p(x, y) \in I_{A}(x, y)$ in white. Information shown in Figures 3 indicates that many pixels fall in the undefined and ambiguous cases. Therefore, assigning a pixel to a class under the highest output criterion is not a recommended criterion. In other analysis it was found that in most cases pixels corresponding to dust storm regions are well defined.

\subsection{C29 ANN Output Adjustment}

A new criterion to determine a winning neuron was defined based on the analysis described in Section 4.3. A wining neuron is the neuron with values greater than 0.6 and with a difference greater than 0.3 with respect the other outputs. The new rule is, Assign pixel $p_{B}$ to class $i$ if $O_{i}>0.6 A N D\left|O_{i}-O_{j}\right|>0.3$ for $i, j=\{v, d, s\} i \neq j$. The color map to describe the new output of the ANN is as indicated in Table 3. Using this new criterion the events were classified again. In most of the cases the results are better because the region of the dust storm is better defined by the new criteria and false positives are reduced as seen in the blue rectangle in Figure 4. The previous process confirm that when undefined outputs and ambiguous outputs are analyzed in the classification processes they positively contribute in the performance of the ANN classifier without negatively effecting the correct detection of the dust storm region. Table 4 shows improvements achieved in the dust storm detection task using the new criteria on the ANN output.

Table 3. Color map of the new ANN outputs

\begin{tabular}{|c|c|c|}
\hline Case & Description & Color \\
\hline Weak output & Less than 0.6 & \\
\hline Undefined & $\begin{array}{l}\text { Outputs with a difference } \\
\text { less than } 0.3\end{array}$ & \\
\hline Both & The two previous cases & \\
\hline
\end{tabular}

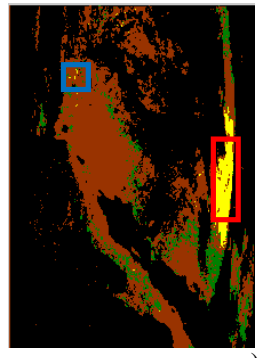

a)

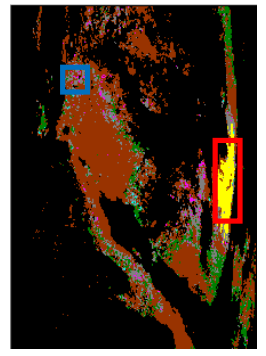

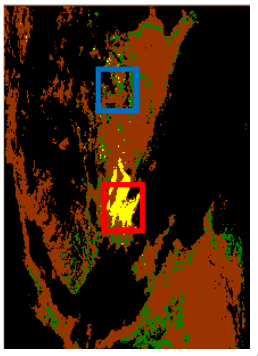

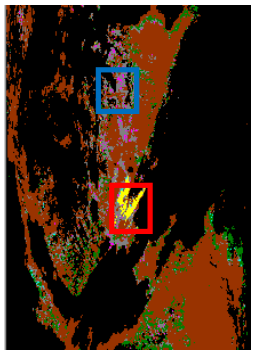

b)

Fig. 4. First result and result with the new criteria for events a) April/6/2001, b) April/10/2001 
Table 4. Improvements with the new criteria on the ANN output

\begin{tabular}{|c|c|c|}
\hline Event & $\begin{array}{l}\text { Problems using the } \\
\text { original criterion }\end{array}$ & $\begin{array}{l}\text { Improvements with } \\
\text { the new criterion }\end{array}$ \\
\hline \multirow[t]{2}{*}{ April/6/2001 } & Disperse dust storm false positives. & Less disperse false positives. \\
\hline & False positives around the dust storm region. & $\begin{array}{l}\text { The dust storm region was better } \\
\text { defined. }\end{array}$ \\
\hline \multirow[t]{2}{*}{ April/10/2001 } & Disperse dust storm false positives. & Less disperse false positives. \\
\hline & False positives around the dust storm region. & $\begin{array}{l}\text { The dust storm region was better } \\
\text { defined. }\end{array}$ \\
\hline July/2/2002 & $\begin{array}{l}\text { The dust storm was not detected. ( the event is } \\
\text { to weak) }\end{array}$ & Same problem. \\
\hline December/17/2002 & Disperse dust storm false positives. & Less disperse false positives. \\
\hline April/15/2003 & There are not problems. & There are not problems. \\
\hline April/15/2003 & False positives around the dust storm region. & $\begin{array}{l}\text { The dust storm region was better } \\
\text { defined. }\end{array}$ \\
\hline \multirow[t]{2}{*}{ April/15/2003 } & Disperse dust storm false positives. & Less disperse false positives. \\
\hline & The dust storm was not detected. & The dust storm was detected. \\
\hline November/22/2003 & Many disperse dust storm false positives. & Less disperse false positives. \\
\hline
\end{tabular}

\section{Results and Conclusions}

Findings in this research indicate that the cloud detection method is a good alternative to reduce the computational burden as well as to get rid of possible perturbations that may cause a negative effect in the performance of the dust storm detector. The ANN classifier presents a suitable performance at detecting the dust storm events. The dust storm events were detected by the method in all events analyzed in this work except in the event \#3 where it was not possible to detect the event. This event is also complicated to be perceived by a human observer. In regard to the false detection rate, we can say that it is tolerable because the cost of the event miss is higher than the false detection error cost. The performance of the ANN classifier is also acceptable, at least under a visual evaluation, at detecting the other two classes, vegetation and soil. The proposed method to manage undefined and ambiguous ANN outputs proved to be an important contribution in this research as the false positives were significant reduced without causing negatives hits in the dust storm area.

In conclusion, the proposed dust storm detector system based on ANN can produce preliminary information related to dust storm detection that may be used for posterior analysis. Moreover, the statistical selection of a relevant training dataset, allowed the construction of a low-complexity ANN model. To overcome uncertainty and ambiguity in ANN outputs, adjustments were made and different criteria were established, which produced higher accuracy rates, as well as a decrease in false positives count. However, false positive issues is an important point that needs more work if the system is intended to be used as a stand-alone dust storm system. 
Acknowledgments. This work was supported by SEP-DGEST, ITCH, UTEP, and partially supported by CONACYT under grant 193324, SEP-DGRI, and Texas Instruments Foundation endowed scholarship.

\section{References}

1. Swapna, J.: Dust Storm Detection Using Classification And Filter Banks. M.S. thesis, University Of Texas At El Paso, El Paso, Texas, USA (2008)

2. Rivera, N.: Detection And Characterization Of Dust Source Area In The Chihuahuan Desert, Southwestern North America. M.S. thesis, University Of Texas At El Paso, El Paso, Texas, USA (2006)

3. Rivas, P., Rosiles, J., Chacon, M.I.: Traditional and Neural Probabilistic Multispectral Image Processing for the Dust Aerosol Detection Problem. In: 2010 IEEE Southwest Symposium on Image Analysis and Interpretation, pp. 169-172 (2010)

4. Qu, J., Xianjun, H., Wang, W., Wang, L., Kafatos, M.: A Study of African Dust Storm and Its Effects on Tropical Cyclones over Atlantic Ocean from Space. In: Geoscience and Remote Sensing Symposium, vol. 4, pp. 2715-2718 (2005)

5. El-Askary, H., Sarkar, S., Kafatos, M.: A Multisensor Approach to Dust Storm Monitoring Over the Nile Delta. IEEE Transactions on Geosciences and Remote Sensing 41, 2386$2391(2003)$

6. San-chao, L., Qinhuo, L., Maofang, G., Liangfu, C.: Detection of Dust Storms by Using Daytime and Nighttime Multi-spectral MODIS Images. In: IEEE International Geosciences and Remote Sensing Symposium, pp. 294-296 (2006)

7. Qu, J., Xianjun, H., Kafatos, M., Wang, L.: Asian Dust Storm Monitoring Combining Terra and Aqua MODIS SRB Measurements. IEEE Geosciences and Remote Sensing Letters, 484-486 (2006)

8. Tao, H., Yaohui, L., Hui, H., Yongzhong, Z., Yujie, W.: Automatic Detection of Dust Storm in the Northwest of China Using Decision Tree Classifier Based on MODIS Visible Bands Data. In: IEEE Geosciences and Remote Sensing Symposium, pp. 3603-3606 (2005)

9. El-Askary, H., Kafatos, M.: Potential for Dust Storm Detection Through Aerosol Radioactive Forcing Related to Atmospheric Parameters. In: Geosciences and Remote Sensing Symposium, pp. 620-623 (2006)

10. Kaufman, Y.J., Tam, D., Dubovik, O., Karnieli, A., Remer, L.A.: Absorption of Sunlight by Dust as Inferred From Satellite and Ground-based Remote Sensing. J. Geophysical Research Letters 28(8), 1479-1482 (2001)

11. Xinping, B., Zhenxin, S.: The Estimation of Dust Aerosol Sources for the Numerical Simulation of Asian Dust Storms Observed in May 2005 in China. In: Geosciences and Remote Sensing Symposium, pp. 828-621 (2006)

12. Huang, J., Minnis, P., Yi, Y., Tang, Q., Wang, X., Hu, Y., Liu, Z., Ayers, K., Trepte, C., Winker, D.: Summer dust aerosols detected from CALIPSO over the Tibetan Plateau. J. Geophysical Research Letters 34, 5 (2007)

13. Kaiping, W., Zhang, T., Bin, H.: Detection of Sand and Dust Storms from MERIS Image Using FE-Otsu Algorithm. In: The 2nd International Conference on Bioinformatics and Biomedical Engineering, pp. 3852-3855 (2008)

14. Ogunjobi, K.O., Kim, Y.J., He, Z.: Aerosol optical properties during Asian dust storm episodes in South Korea. In: Theoretical and Applied Climatology, vol. 76(1-2), pp. 6575. Springer Wien, Heidelberg (2004)

15. El-Askary, H., Abhishek, A., El-Ghazawi, T., Menas, K., Le-Moigne, J.: Enhancing Dust Storm Detection Using PCA based Data Fusion. In: Geoscience and Remote Sensing Symposium, pp. 1424-1427 (2005) 